\title{
Transport and Non-Invasive Position Detection of Electron Beams from Laser-Plasma Accelerators
}

\author{
J. Osterhoff*, T. Sokollik*, , K. Nakamura*, M. Bakeman*, \\ R. Weingartner**, , A. J. Gonsalves*, S. Shiraishi*, C. Lin*, J. van Tilborg*, C. G. R. \\ Geddes*, C. B. Schroeder*, E. Esarey*, Cs. Tóth*, S. De Santis*, \\ J. M. Byrd*, F. Grüner*** and W. P. Leemans* \\ *Lawrence Berkeley National Laboratory, Berkeley, CA 94720, USA \\ ${ }^{\dagger}$ University of California, Berkeley, CA 94720, USA \\ **Ludwig-Maximilians-Universität München, 85748 Garching, Germany \\ ${ }^{\ddagger}$ Max-Planck-Institut für Quantenoptik, 85748 Garching, Germany
}

\begin{abstract}
The controlled imaging and transport of ultra-relativistic electrons from laser-plasma accelerators is of crucial importance to further use of these beams, e.g. in high peak-brightness light sources. We present our plans to realize beam transport with miniature permanent quadrupole magnets from the electron source through our THUNDER undulator. Simulation results demonstrate the importance of beam imaging by investigating the generated XUV-photon flux. In addition, first experimental findings of utilizing cavity-based monitors for non-invasive beam-position measurements in a noisy electromagnetic laser-plasma environment are discussed.
\end{abstract}

Keywords: Electron-beam transport, laser-plasma acceleration, permanent magnet quadrupole, beam-position monitor PACS: $52.38 . \mathrm{Kd}, 41.75 . \mathrm{Jv}, 41.85 . \mathrm{Lc}$

\section{INTRODUCTION}

The field of laser-plasma acceleration (LPA) of electrons in plasma has made tremendous progress in recent years and the quality and stability of produced electron beams has been steadily improved [1,2,3]. However, some key properties of LPA-generated beams such as their pointing stability and divergence cannot rival the quality of electron bunches from conventional accelerators, yet. This may lead to complications and reproducibility issues when such beams are used for actual applications, e.g. as drivers for undulator-based photon sources $[4,5]$ or in the future for free-electron lasers (FELs) [6]. Therefore, an enhanced control over beam divergence, size, pointing direction and position, i.e. transverse phase-space, are of fundamental importance for the applicability of electron beams from a laser-plasma accelerator.

Beam phase-space manipulation can be achieved by propagating electron beams through magnetic multipole fields, in which dipole field components allow for beam steering, quadrupole components for chromatic beam focusing, sextupole fields for chromaticity compensation, etc. Traditionally, in conventional radio-frequency accelerator facilities these magnetic fields are generated by electro-magnetic coils, which are typically bulky and in case of quadrupole fields achieve gradients on the order of $10 \mathrm{~T} / \mathrm{m}$. In order to deploy such magnets for beam focusing in an LPA setup with characteristic energies of several hundred $\mathrm{MeV}$ and focusing distances on the order of a few meters, the magnetic quadrupole lenses would need to have lengths on the order of several ten centimeters. These dimensions allow for electron trajectory path differences, which will ultimately result in a significant temporal elongation of femtosecond electron beams [7]. Owing to the inherently short duration of laser-plasma accelerated beams on a scale of $\sim 10 \mathrm{fs}$, small magnetic quadrupole lenses with gradients on the order of several hundred $\mathrm{T} / \mathrm{m}$ are required for conservation of the initial temporal pulse structure.

Field gradients of this magnitude can be readily achieved with miniature permanent magnet quadrupoles (PMQs) $[8,9]$. In the following section, we report on the implementation of such lenses into the THUNDER undulator [10] beamline based at the LOASIS program of the Lawrence Berkeley National Laboratory and discuss the consequences of a controlled electron beam transport for the upcoming undulator experiments. Moreover, in the second part of this paper, a first demonstration of a non-invasive measurement of the position of laser-accelerated electron beams with a cavity-based beam position monitor (BPM) will be presented. Knowledge of beam position and direction will 

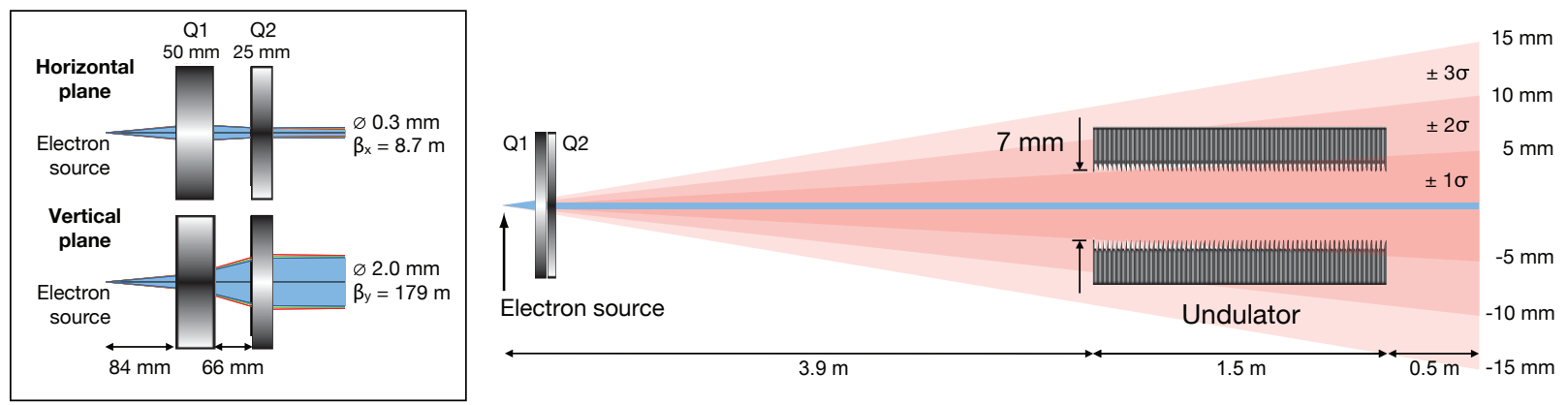

FIGURE 1. Design study of electron beam transport by a PMQ doublet from the electron source through an undulator. The beam envelope of a freely diverging beam with a divergence opening angle $\sigma=1 \mathrm{mrad} \mathrm{rms}, 500 \mathrm{MeV}$ in energy, and normalized emittance $1 \mathrm{~mm} \cdot \mathrm{mrad}$ is shown in different shades of red for $\pm 1,2$, and $3 \sigma$. The imaged envelope of the same beam is depicted in blue. The inset displays detailed quadrupole positions and additional beam parameters.

be essential not only for the alignment of the PMQs but also for the interpretation of detected photon spectra from THUNDER for typical LPA beam pointing fluctuations of $\sim 1 \mathrm{mrad}$ rms from the electron source.

\section{ELECTRON BEAM TRANSPORT}

An electron beam transport system based on quadrupole magnets has to consist of at least two lenses. Each lens will focus the electron beam in one plane and defocus it in the perpendicular plane. Therefore a combination of two lenses with one being rotated with respect to the other by $90^{\circ}$ around the axis of beam propagation may allow for a total, albeit astigmatic focusing effect in both planes. Since the magnetic field gradients and lengths of the utilized PMQs are fixed, the focal strength of the system may be changed by varying the distances between the electron source, the first lens, and the second lens.

Fig. 1 displays a schematic of the electron beam transport through the THUNDER undulator. The drawn beam envelopes for the freely diverging case show that without a transport system even a beam typical for state-of-theart laser-plasma accelerators with a low divergence of $1 \mathrm{mrad} \mathrm{rms}$ will not propagate through the undulator without scraping its surface, possibly causing demagnetization of the device. This problem will become even more severe when characteristic pointing fluctuations of $\sim 1 \mathrm{mrad} \mathrm{rms}$ are considered. Thus, it is clear that a beam transport system will be required, which collimates the electron beam and mitigates the influence of variations in pointing direction. The two utilized PMQs (design described in [9]) of inner diameter $5 \mathrm{~mm}$ with field gradients of $503 \mathrm{~T} / \mathrm{m}$ over a length of $50 \mathrm{~mm}$ and $490 \mathrm{~T} / \mathrm{m}$ over $25 \mathrm{~mm}$, respectively, are positioned at specific distances (as given in Fig. 1) from the electron beam source, so the source may be imaged into the center of the undulator with waist sizes in each dimension much smaller than the undulator gap and $\beta$-functions large compared to the undulator length. Those distances were found by maximizing $\beta$ using the beam propagation code COSY INFINITY [11] for LPA-accelerated electron beams of $500 \mathrm{MeV}$ energy, $1 \% \mathrm{rms}$ energy spread and $1 \mathrm{~mm} \cdot \mathrm{mrad}$ normalized emittance, emerging with $1 \mathrm{mrad}$ initial divergence from the source. An additional requirement that places constraints on the positioning of the lenses arises from the fact that clipping of both the electron beam and laser pulse at the PMQs should be omitted to prevent damage.

An electron beam sent through the undulator with proper mode matching will result in a detected photon yield from the THUNDER undulator that is a factor $\sim 50$ greater than an unmatched beam (cf. Fig. 2). This has been computed with the synchrotron radiation code SPECTRA [12] for parameters mirroring the undulator setup (undulator parameter $K=1.25$, period length $\lambda_{u}=21.8 \mathrm{~mm}, 66$ periods), and taking into account a slit of $15 \mu \mathrm{m}$ width in front of the XUV-spectrometer detecting the undulator radiation [13]. The enhanced yield can be explained by the following mechanisms: owing to the decreased beam divergence, the variation in electron propagation direction is greatly reduced, leading to the fact that the XUV detector is observing photon emission under similar angles for most electrons. Since the measured emission wavelength $\lambda$ depends on the observation angle $\Theta$ through

$$
\lambda=\frac{\lambda_{u}}{2 N \gamma^{2}}\left(1+\frac{K^{2}}{2}+\gamma^{2} \Theta^{2}\right)
$$




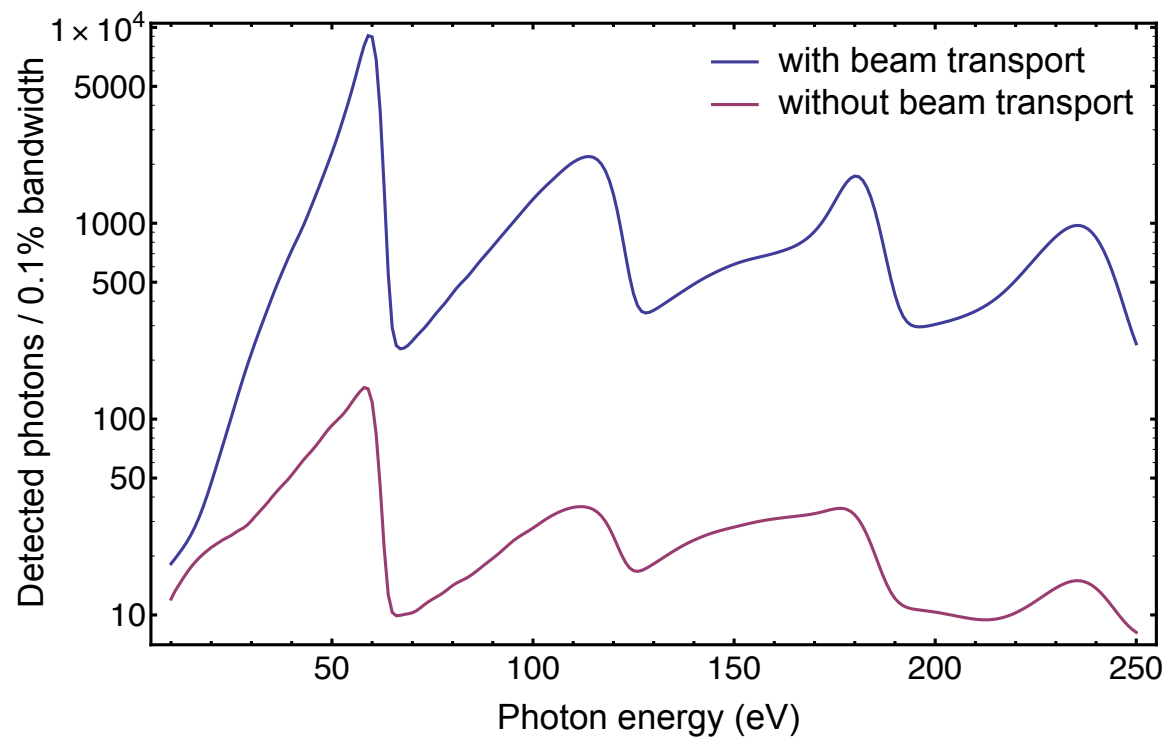

FIGURE 2. Theoretical comparison of the detected XUV-photon spectrum with and without electron beam transport by PMQs. The results were obtained from the synchrotron radiation code SPECTRA [12] and show an increase of $\sim 50$ in the detected photon numbers for the transported beam.

with $N$ being the harmonic number and $\gamma$ being the relativistic factor, the number of photons per unit bandwidth grows. Furthermore, a larger number of electrons will actually contribute to the measured spectrum and propagate through the undulator in the imaged case, whereas in the free-propagation scenario, electrons can hit the undulator surface and are lost. The latter process is not included in the presented simulation. Hence the 50-fold increase in XUV-signal constitutes a conservative estimate.

\section{BEAM POSITION DIAGNOSTIC}

For the interpretation of the recorded XUV-spectra and for their proposed use as a single-shot diagnostic of electron emittance and energy spectrum [14], it is important to know the electron beam trajectory through the undulator as accurately as possible due to the $\Theta$ dependence of $\lambda$ (see Eq. (1)) and due to spatial inhomogeneities in $K$. For this reason, it is desirable to measure the position and propagation angle of electron bunches behind the imaging quadrupole doublet and before they enter the undulator. To date, beam position measurements in LPAs have almost exclusively been conducted using phosphor screens. In addition, some first measurements of optical transition radiation have been reported for that purpose [15]. However, both of these techniques have in common that electron beams need to traverse matter, which leads to scattering of the beam and deteriorates its emittance. For future applications of laser-accelerated electrons in emittance-sensitive applications such as FELs or for the undulator-based emittance diagnostic mentioned above, non-invasive position diagnostics will become mandatory. In this section, the first use of a non-invasive cavitybased beam position monitor in an LPA-beamline will be discussed.

The deployed BPM $[16,17]$ consists of a microwave resonator, which can be excited by an electron beam passing through. Its cavity primarily supports the $\mathrm{TM}_{110}$ dipole mode and exhibits a slightly elliptical shape, which consequently leads to different resonance frequencies of $2.85 \mathrm{GHz}$ and $2.86 \mathrm{GHz}$ in the horizontal and vertical plane, respectively. Two pick-up ports for each plane located at opposite sites of the cavity allow for monitoring four ringdown signals (see example in Fig. 3a). Their envelope functions and signal phases are extracted after digital down conversion (cf. Fig. 3b). The amplitude $A$ of an envelope signal is proportional to the charge $q$ of the electron beam and its relative distance from the cavity center $x$, whereas the phase determines the sign of the signal, e.g. the side of electron-beam passage relative to the cavity center. It is important to note that such a BPM by itself cannot be used for an accurate position measurement, since $A$ needs to be normalized to $q$ in order to obtain $x$. Thus, an independent non-invasive charge measurement by e.g. an integrating current transformer or a monopole mode cavity should be 

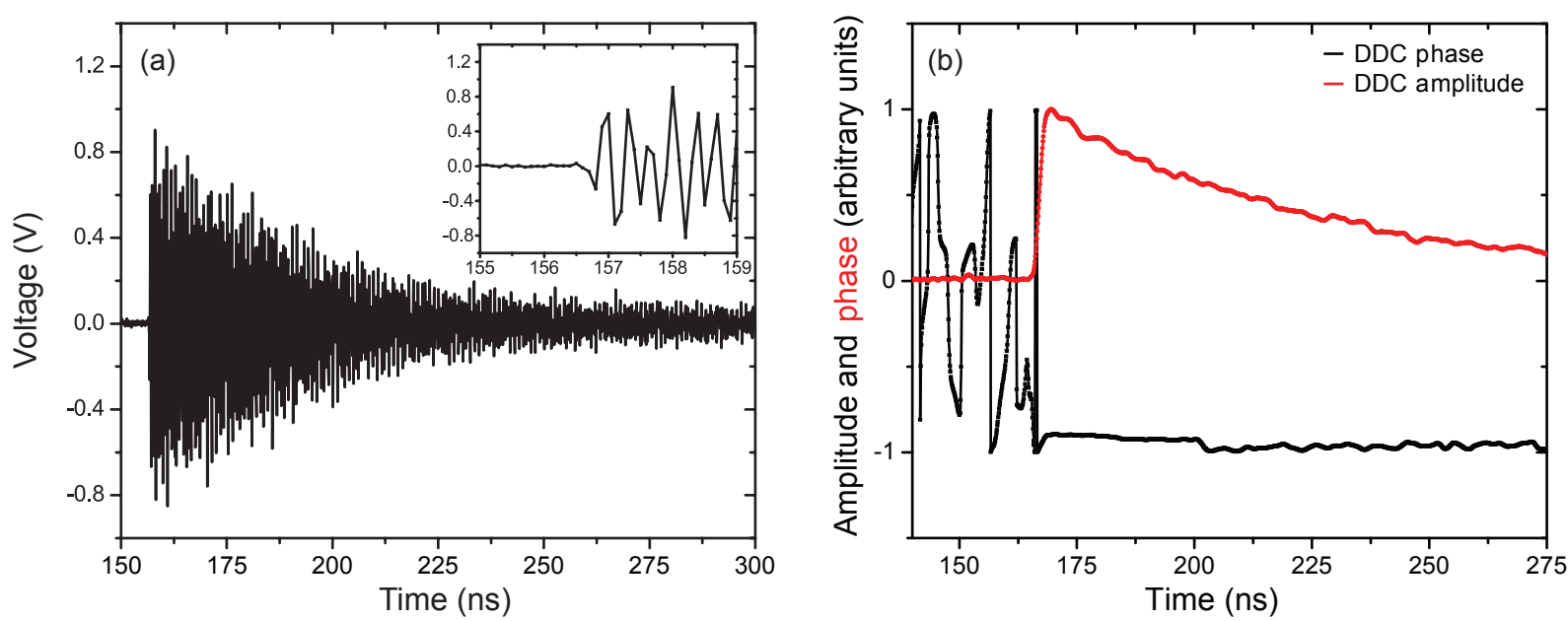

FIGURE 3. Data from a single BPM channel: (a) Raw ring-down data. The inset shows a magnification of the first oscillations. (b) The obtained amplitude (black) and phase (red) after digital down conversion.
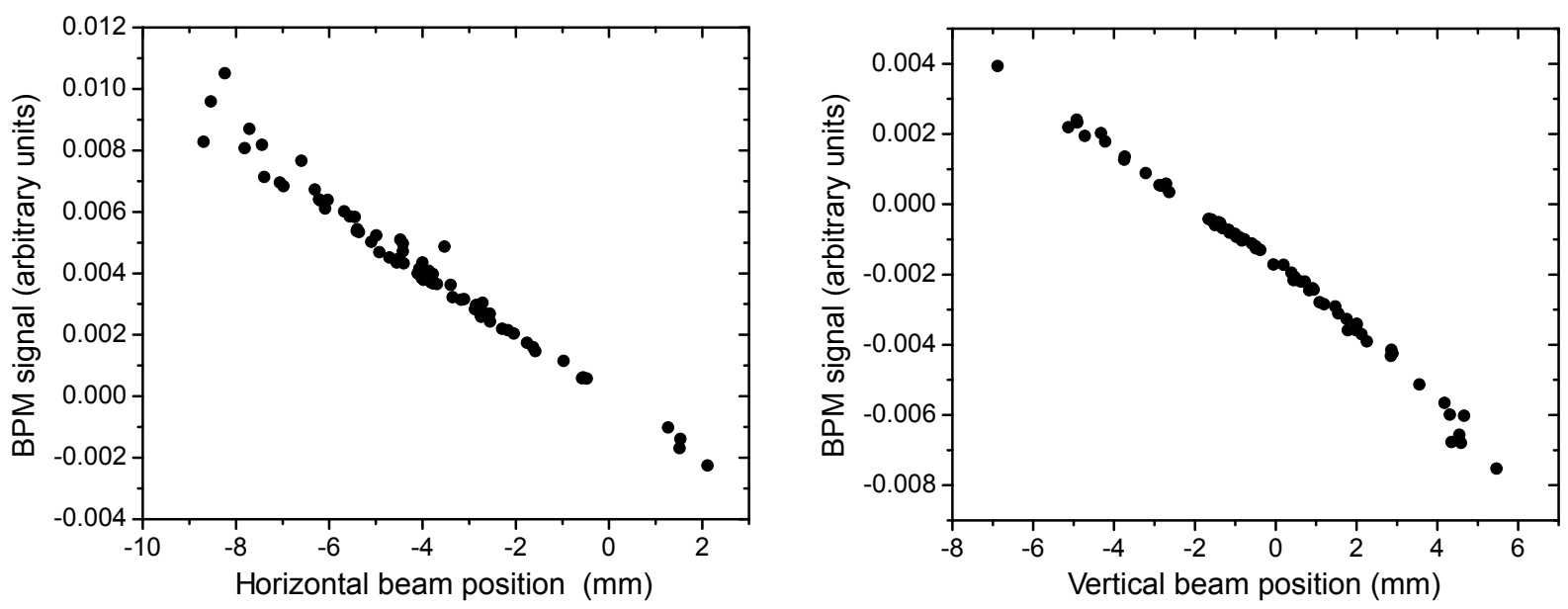

FIGURE 4. Correlation between the BPM signal and the measured electron beam position from a downstream phosphor screen in the horizontal (left plot) and vertical (right plot) planes.

performed simultaneously. For the sake of simplicity, a calibrated phosphor screen mounted $\sim 0.5 \mathrm{~m}$ downstream of the BPM enabled the charge detection and position cross-calibration in this proof-of-principle experiment.

The recorded electron bunches were generated in an LPA featuring a broadband spectral distribution with quasimonoenergetic components of peak energies up to $\sim 250 \mathrm{MeV}$ and an average beam charge of $58.7 \pm 11.1 \mathrm{pC}$. The correlation between beam positions from phosphor screen and BPM are shown in Fig. 4. As expected, a unique linear dependence may be seen. The accuracy of this measurement is limited to sub-millimeter by the finite spatial and charge resolution of the phosphor screen. The cavity BPM itself has been shown to support sub-micrometer accuracies [16]. Additional tests demonstrated that this type of BPM is sufficiently sensitive to detect electron bunches of only few pC charge. Furthermore it proved largely insensitive to electromagnetic noise originating from a $25 \mathrm{kV}$-plasma discharge and from the laser-plasma interaction itself. 


\section{CONCLUSIONS}

In summary, the application of miniature magnetic quadrupole lenses will allow for the manipulation of transverse phase-space properties of laser-plasma accelerated electron beams. Moreover, cavity-based BPMs have been utilized to measure electron beam position non-invasively, while being insensitive to the noisy electromagnetic environment in laser-plasma accelerators. Both types of devices will prove important and potentially indispensable for future applications of such beams in free-electron lasers and single-shot undulator-based emittance diagnostics.

\section{ACKNOWLEDGMENTS}

We would like to thank Zachary Wolf and his team at SLAC for assistance in characterizing the PMQs. Furthermore, the PMQ development was supported by the DFG-Cluster of Excellence Munich-Centre for Advanced Photonics (MAP). This work was supported by the Director, Office of Science, Office of High Energy Physics, of the U.S. Department of Energy under Contract No. DE-AC02-05CH11231, and DTRA.

\section{REFERENCES}

1. W. P. Leemans, B. Nagler, A. J. Gonsalves, C. Tóth, K. Nakamura, C. G. R. Geddes, E. Esarey, C. B. Schroeder, and S. M. Hooker, Nature Physics 2, 696-699 (2006).

2. J. Faure, C. Rechatin, A. Norlin, A. Lifschitz, Y. Glinec, and V. Malka, Nature 444, 737-739 (2006).

3. J. Osterhoff, A. Popp, Z. Major, B. Marx, T. P. Rowlands-Rees, M. Fuchs, M. Geissler, R. Hörlein, B. Hidding, S. Becker, E. A. Peralta, U. Schramm, F. Grüner, D. Habs, F. Krausz, S. M. Hooker, and S. Karsch, Physical Review Letters 101, 085002 (2008).

4. H.-P. Schlenvoigt, K. Haupt, A. Debus, F. Budde, O. Jäckel, S. Pfotenhauer, H. Schwoerer, E. Rohwer, J. G. Gallacher, E. Brunetti, R. P. Shanks, S. M. Wiggins, and D. A. Jaroszynski, Nature Physics 4, 130-133 (2008).

5. M. Fuchs, R. Weingartner, A. Popp, Z. Major, S. Becker, J. Osterhoff, I. Cortrie, B. Zeitler, R. Hörlein, G. D. Tsakiris, U. Schramm, T. P. Rowlands-Rees, S. M. Hooker, D. Habs, F. Krausz, S. Karsch, and F. Grüner, Nature Physics 5, 826-829 (2008).

6. F. Grüner, S. Becker, U. Schramm, T. Eichner, M. Fuchs, R. Weingartner, D. Habs, J. Meyer-ter Vehn, M. Geissler, M. Ferrario, L. Serafini, B. van der Geer, H. Backe, W. Lauth, and S. Reiche, Applied Physics B: Lasers and Optics 86, 431-435 (2007).

7. R. Weingartner, M. Fuchs, A. Popp, S. Raith, S. Becker, S. Chou, M. Heigoldt, K. Khrennikov, J. Wenz, B. Zeitler, Z. Major, J. Osterhoff, F. Krausz, S. Karsch, and F. Grüner, "Imaging laser-wakefield-accelerated electrons using miniature magnetic quadrupole lenses", Physical Review Special Topics - Accelerators and Beams (submitted).

8. J. K. Lim, P. Frigola, G. Travish, J. B. Rosenzweig, S. G. Anderson, W. J. Brown, J. S. Jacob, C. L. Robbins, and A. M. Tremaine, Physical Review Special Topics - Accelerators and Beams 8, 072401 (2005).

9. T. Eichner, F. Grüner, S. Becker, M. Fuchs, D. Habs, R. Weingartner, U. Schramm, H. Backe, P. Kunz, and W. Lauth, Physical Review Special Topics - Accelerators and Beams 10, 082401 (2007).

10. K. Robinson, D. Quimby, and J. Slater, IEEE Journal of Quantum Electronics 23, 1497-1513 (1987).

11. K. Makino, and M. Berz, Nuclear Instruments and Methods in Physics Research Section A: Accelerators, Spectrometers, Detectors and Associated Equipment 558, 346-350 (2006).

12. T. Tanaka, and H. Kitamura, Journal of Synchrotron Radiation 8, 1221-1228 (2001).

13. M. S. Bakeman, J. van Tilborg, T. Sokollik, D. Baum, N. Ybarrolaza, R. Duarte, C. Tóth, and W. P. Leemans, Review of Scientific Instruments (accepted for publication).

14. M. S. Bakeman, C. B. Schroeder, K. E. Robinson, C. Tóth, K. Nakamura, W. M. Fawley, and W. P. Leemans, AIP Conference Proceedings 1086, 643-648 (2009).

15. C. Lin, K. Nakamura, A. J. Gonsalves, J. van Tilborg, T. Sokollik, J. Osterhoff, S. Shiraishi, C. Benedetti, C. B. Schroeder, E. Esarey, and W. P. Leemans, AIP Conference Proceedings (in this issue).

16. S. Walston, S. Boogert, C. Chung, P. Fitsos, J. Frisch, J. Gronberg, H. Hayano, Y. Honda, Y. Kolomensky, A. Lyapin, S. Malton, J. May, D. McCormick, R. Meller, D. Miller, T. Orimoto, M. Ross, M. Slater, S. Smith, T. Smith, N. Terunuma, M. Thomson, J. Urakawa, V. Vogel, D. Ward, and G. White, Nuclear Instruments and Methods in Physics Research Section A: Accelerators, Spectrometers, Detectors and Associated Equipment 578, 1-22 (2007).

17. M. Slater, C. Adolphsen, R. Arnold, S. Boogert, G. Boorman, F. Gournaris, M. Hildreth, C. Hlaing, F. Jackson, O. Khainovski, Y. G. Kolomensky, A. Lyapin, B. Maiheu, D. McCormick, D. J. Miller, T. J. Orimoto, Z. Szalata, M. Thomson, D. Ward, M. Wing, and M. Woods, Nuclear Instruments and Methods in Physics Research Section A: Accelerators, Spectrometers, Detectors and Associated Equipment 592, 201-217 (2008). 
DISCLAIMER: This document was prepared as an account of work sponsored by the United States Government. While this document is believed to contain correct information, neither the United States Government nor any agency thereof, nor The Regents of the University of California, nor any of their employees, makes any warranty, express or implied, or assumes any legal responsibility for the accuracy, completeness, or usefulness of any information, apparatus, product, or process disclosed, or represents that its use would not infringe privately owned rights. Reference herein to any specific commercial product, process, or service by its trade name, trademark, manufacturer, or otherwise, does not necessarily constitute or imply its endorsement, recommendation, or favoring by the United States Government or any agency thereof, or The Regents of the University of California. The views and opinions of authors expressed herein do not necessarily state or reflect those of the United States Government or any agency thereof or The Regents of the University of California. 